\title{
La investigación formativa en las prácticas docentes de los profesores de un programa de contaduría pública*
}

doi:10.11144/Javeriana.cc17-43.ifpd

\section{William Rodrigo Avendaño-Castro \\ Doctor (c) en ciencias sociales y humanas, Universidad Javeriana, Bogotá, Colombia. Magíster (c) en educación, Universidad de La Sabana, Bogotá. Magíster en comercio internacional, Prime Business School, Universidad Sergio} Arboleda, Bogotá. Magíster en administración, Universidad Santo Tomás, Bucaramanga, Colombia. Especialista en comercio internacional, Georgetown University, Universidad Sergio Arboleda, Bogotá. Especialista en alta gerencia, Universidad Libre, Cúcuta, Colombia. Economista, Universidad Los Libertadores, Bogotá. Profesor-investigador asociado, adscrito al Departamento de Estudios Internacionales y de Fronteras, Facultad de Ciencias Empresariales, Universidad Francisco de Paula Santander. Director del grupo de investigación en Ciencias Sociales y Humanas, GICSH, adscrito a la Facultad de Ciencias Empresariales, Universidad Francisco de Paula Santander, Cúcuta.

Correo electrónico: williamavendano@ufps.edu.co

\section{Gerson Rueda-Vera}

Doctorando en educación, Universidad Pedagógica Experimental Libertador, UPEL, Rubio, Venezuela. Magíster en práctica pedagógica, Universidad Pedagógica Experimental Libertador, UPEL, Rubio, Venezuela. Contador público, Universidad Francisco de Paula Santander, Cúcuta,
Colombia. Profesor adscrito a la Facultad de Ciencias Empresariales, Universidad Francisco de Paula Santander, Cúcuta, Colombia. Docente-investigador, miembro del grupo de investigación en Ciencias Sociales y Humanas, GICSH, adscrito al Departamento de Estudios Internacionales y Fronteras, Facultad de Ciencias Empresariales, Universidad Francisco de Paula Santander, Cúcuta, Colombia. Correo electrónico: gersonruedavera@ufps.edu.co

\section{Luisa Stella Paz-Montes}

Candidata a doctor en educación, Universidad Pedagógica Experimental Libertador, UPEL, Rubio, Venezuela. Magíster en gerencia de empresas, Universidad Experimental del Táchira, San Cristóbal, Venezuela. Especialista en gerencia educativa, Universidad Católica de Manizales, Manizales, Colombia. Administradora de empresas, Universidad Francisco de Paula Santander, Cúcuta, Colombia. Profesora adscrita a la Facultad de Ciencias Empresariales, Universidad Francisco de Paula Santander. Docente-investigadora, miembro del grupo de investigación en Ciencias Sociales y Humanas, GICSH, adscrito al Departamento de Estudios Internacionales y Fronteras, Facultad de Ciencias Empresariales, Universidad Francisco de Paula Santander, Cúcuta, Colombia.

Correo electrónico: luisastellapm@ufps.edu.co

\footnotetext{
* Artículo de investigación formativa
} 
Resumen El artículo analiza el papel y la dinámica de la investigación formativa en las prácticas docentes de un grupo de profesores adscritos al Programa de Contaduría Pública de la Universidad Francisco de Paula Santander, de la ciudad de Cúcuta, Colombia. Para el cumplimiento del objetivo, se hace un estudio empírico-analítico de naturaleza correlacional con una muestra de 60 docentes del programa de contaduría pública. Se utiliza una encuesta estructurada, la cual fue sometida a validez de contenido por pares expertos y a confiabilidad estadística. Los resultados demuestran 75 relaciones significativas de un total de 144. En conclusión, los sujetos que participan en el estudio demuestran que pocas prácticas docentes se realizan en función de la investigación formativa.

Palabras clave Investigación formativa; investigación productiva; prácticas docentes; prácticas pedagógicas; contaduría pública

Códigos JEL I2, I29, M4, M49

\section{Educational Research in Teaching Practices of Teachers of a Public Accounting Program}

\begin{abstract}
This article analyzes the role and dynamics of educational research in the teaching practices of a group of teachers affiliated to the Public Accounting Program of the Universidad Francisco de Paula Santander, in Cucuta, Colombia. In order to meet the objective we carried out an empirical-analytic study, correlational in nature, with a sample of 60 teachers of the public accounting program. We used a structured survey subject to content validation with expert peers and statistical reliability. Results show 75 significant relationships out of a total of 144 . In conclusion, subjects participating in the study showed few teaching practices are carried out driven towards educational research.
\end{abstract}

Keywords Educational research; productive research; teaching practices; educational practices; public accounting

\section{A pesquisa formativa nas práticas docentes dos professores de um programa de contabilidade pública}

Resumo O artigo analisa o papel e dinâmica da pesquisa formativa nas práticas docentes de um grupo de professores adscritos ao Programa de Contabilidade Pública da Universidade Francisco de Paula Santander, da cidade de Cúcuta, Colômbia. Para cumprir o objetivo, estudo empírico-analítico de natureza correlacional foi feito com amostra de 60 docentes do programa de contabilidade pública. Utilizou-se inquérito estruturado, que foi submetido a validade de conteúdo por pareceristas expertos e a confiabilidade estadística. Os resultados mostram 75 relações significativas de um total de 144. Em conclusão, os sujeitos que participam no estudo mostram que poucas práticas docentes são feitas em função da pesquisa formativa.

Palavras-chave Pesquisa formativa; pesquisa produtiva; práticas docentes; práticas pedagógicas; contabilidade pública

\section{Introducción}

Una de las principales características de la actual sociedad del conocimiento es la de estar centrada en la capacidad de las personas y grupos académicos para identificar, sistematizar, analizar, producir, socializar y transferir saberes. En una sociedad de este tipo, la investigación es una de las principales actividades pues de ella depende la posibilidad de que los sujetos puedan elaborar y reelaborar el conocimiento. En este orden, la universidad se convierte en el espacio sociocultural institucionalizado para enseñar a investigar -investigación formativa-y hacer investigación -investigación productiva-(Restrepo- 
LA INVESTICACIÓN FORMATIVA EN LAS PRÁCtiCAS DOCENTES / W. AVENDAÑo, G. RUEDA, L. PAZ / 159

Gómez, 2003; Ruiz-Bolívar \& Torres-Pacheco, 2005; Sancho-Gil, 2001).

El término investigación formativa hace referencia a la formación en y para la investigación. Es el conjunto de acciones orientadas a favorecer la apropiación y el desarrollo de los conocimientos, las habilidades y las actitudes necesarias para que estudiantes y profesores puedan desempeñar con éxito actividades productivas asociadas a la investigación científica, el desarrollo tecnológico y la innovación, ya sea en el sector académico o en el productivo (Guerrero-Useda, 2007). Por otro lado, Bernardo Restrepo-Gómez (2003) propone el término investigación productiva para referirse a la investigación científica en sentido estricto, a la producción o generación sistemática de conocimiento y a su aplicación para resolver problemas del contexto.

La calidad de la educación ofertada por las universidades está íntimamente ligada con la capacidad de estas para instruir a los futuros profesionales en el campo de la investigación, usar efectivamente la investigación en la mejora de los procesos de enseñanza, y planear y desarrollar investigaciones que permitan la generación de nuevo conocimiento y el avance tecnológico (Cerda-Gutiérrez, 2007). La investigación y la producción de conocimiento de mayor impacto están asociadas con el nivel de las universidades y su capacidad para establecer puentes de comunicación y ayuda con otras instituciones de educación superior (Jones, Wuchty \& Uzzi, 2008), la efectividad de las estrategias para involucrar en las investigaciones a las comunidades y facilitar la participación de los grupos sociales (Weerts
\& Sandmann, 2010), y las habilidades para transferir eficazmente el conocimiento a partir de la disponibilidad de los recursos institucionales, organizacionales, humanos y tecnológicos (Hewitt-Dundas, 2012).

Hay una posición consensuada entre académicos y expertos en cuanto al papel fundamental que cumple la universidad en la socialización y fortalecimiento de las actividades investigativas, lo cual le permite, entre otras cosas, adoptar una posición autocrítica frente a los saberes producidos y reproducidos. Para Bernardo Restrepo-Gómez (2003), la investigación en la universidad corresponde a un "proceso de búsqueda de nuevo conocimiento, proceso caracterizado por la creación del acto, por la innovación de ideas, por los métodos rigurosos utilizados, por la autocrítica y por la validación y juicio crítico de pares" (p. 196). En otras palabras, la investigación sustenta el espíritu de las universidades en la medida en que les permite proyectarse en el ámbito social, cultural, político y económico, dando sentido al conocimiento producido y a los saberes transferidos a partir del diálogo válido entre pares.

El contexto específico de las actuales universidades está marcado al menos por dos procesos: la globalización y la sociedad del conocimiento. Estos implican para las universidades una mayor dinámica en términos de actividad investigativa y, en consecuencia, las prácticas de los docentes deben estar fuertemente afianzadas con la investigación tanto formativa como productiva. Para María Eugenia Guerrero-Useda (2007), la educación superior cuenta con un rol estratégico en "la generación de conocimientos e innovaciones tecnológicas, [y] 
en la formación de profesionales capaces de estudiar y aportar a la comprensión y solución de los problemas del entorno" (p. 190). Entonces, uno de los desafíos más importantes para los docentes adscritos a la educación superior es producir conocimiento en las diversas áreas científicas y propender con las prácticas pedagógicas a la formación de sujetos críticos con carácter científico.

En América Latina, una de las problemáticas más relevantes para las universidades es la poca efectividad de la formación investigativa (Hurtado de Barrera, 2002), es decir, en la instrucción de estudiantes en materia de investigación que les permita explorar nuevos enfoques y teorías aplicadas a escenarios diversos. Este problema está asociado a la dificultad de muchos docentes universitarios para actuar en un marco de complejidad e interdisciplinariedad, en el cual los saberes socializados sean el producto de un diálogo abierto, diversificado y con carácter científico. Marina Yolanda Gutiérrez y Guadalupe Langarica (1994) sostienen que es necesario formar grupos de investigadores interdisciplinarios que permitan la reflexión profunda sobre cada campo de estudio en un marco de autonomía e independencia académica. De este modo, "las universidades deben promover cambios estructurales para reducir las barreras de investigación que limitan la labor de investigadores en múltiples disciplinas" (Sá, 2008, p. 537).

La investigación (formativa y productiva) y la docencia son indicadores imprescindibles para evaluar la calidad de la educación superior. En el caso particular de la investigación productiva, el contenido y la configuración de redes es lo más importante pues a partir de ello se puede establecer el impacto de la actividad investigativa de las universidades (Bello, 2003). En cuanto a la investigación formativa, se busca que los estudiantes al menos: (1) generen conocimientos con rigurosidad científica y contextualizados en su campo del saber, (2) ayuden en la resolución de problemas, (3) realicen diagnósticos pertinentes de los fenómenos, (4) complementen sus saberes desde otras perspectivas y enfoques, (5) analicen e interpreten datos e información propia de su campo, y (6) mejoren sus procesos de escritura académica a través de la producción de informes técnico-científicos (Ruiz-Bolívar \& Torres-Pacheco, 2015, p. 15).

En Colombia, el Decreto 1295 de 2010 (Colombia, 2010) establece las exigencias formales a las instituciones de educación superior en materia de formación investigativa, con el propósito de generar una cultura que permita el impulso de la investigación productiva, la construcción de un pensamiento crítico y autónomo, y la generación y transferencia de nuevos conocimientos. El Decreto en particular señala que para la obtención del registro calificado, es decir, la acreditación que valida el cumplimiento de las condiciones mínimas necesarias para la oferta y desarrollo de programas académicos, la universidad debe diseñar e implementar actividades de investigación que les permita a los estudiantes adquirir una actitud crítica y una capacidad innovadora en torno a las ciencias, disciplinas, tecnologías, artes y/o humanidades. Y para ello se verifican (1) los modos en que desarrolla la investigación formativa, (2) los procedimientos para la incorporación de 
LA INVESTIGACIÓN FORMATIVA EN LAS PRÁCticas DOCENTES / W. AVENDAÑo, G. RUEDA, L. PAZ / 161

las TIC, (3) el ambiente apropiado para la innovación y la creatividad, (4) un direccionamiento estratégico que promocione la investigación, (5) los productos de investigación, (6) la disponibilidad de docentes para la investigación formativa y productiva, (7) las relaciones con el sector externo en cuanto al desarrollo de investigaciones.

Para las universidades, la implementación y el logro de los objetivos trazados en el Decreto en mención implican verdaderos retos que van desde la gestión de recursos hasta el cambio de la cultura universitaria, y en ello cumplen un rol fundamental el currículo, el ambiente universitario, los semilleros de investigación y el apoyo a la investigación (Bocanegra, 2006, p. 7).

Por lo anterior, se espera que cualquier programa de formación universitaria cuente con las características y particularidades mínimas exigidas en términos de investigación formativa y productiva. En la investigación formativa, el requerimiento esencial son las prácticas docentes y pedagógicas, pues no hay educación sin acto humano mediador. A partir de sus prácticas pedagógicas, los docentes pueden fortalecer y potenciar la efectividad de los procesos de enseñanza-aprendizaje y convertir las prácticas en la más destacada herramienta del docente para llevar a cabo su función socioeducativa. La primera etapa para impulsar un cambio en esta dimensión, evaluar las prácticas pedagógicas de los docentes, a fin de establecer los avances y retos que se presentan y, con ello, tomar decisiones para la mejora de la investigación formativa.

En este artículo se analiza el papel y la dinámica de la investigación formativa en las prácticas docentes de un grupo de profesores adscritos al programa de contaduría pública de la Universidad Francisco de Paula Santander, de la ciudad de Cúcuta, Colombia. Como se ha descrito hasta el momento, la investigación productiva depende en gran medida de la investigación formativa y, por ende, de las prácticas docentes que se diseñan e implementan con los estudiantes. En el programa de contaduría pública de la Universidad Francisco de Paula Santander, las prácticas docentes no han permitido una buena gestión y desarrollo de la investigación formativa; este fenómeno exige un análisis profundo con el propósito de reconocer sus características y las limitaciones de este proceso.

\section{La investigación formativa: acercamiento conceptual}

Los docentes universitarios se ven involucrados de forma permanente con la investigación en todas sus tareas y actividades. Ken Bain (2007), al describir las prácticas pedagógicas de profesores universitarios, señala que el éxito docente se basa en un amplio conjunto de características en las que se destacan el amplio manejo y el conocimiento del área de estudio; la capacidad para el manejo de un saber interdisciplinar; la habilidad para la planeación, ejecución y evaluación de las clases a partir de aquello que necesitan los estudiantes; la facultad para potenciar habilidades u operaciones mentales en los estudiantes y que posibilitan el tratamiento de la información, entre otras. Estas características están profundamente ligadas con el proceso de investigación pues solo un docente que participa activamente en el ámbito investigativo 
logra poseer, socializar y transferir el conocimiento de una manera efectiva.

En conclusión, la investigación es entendida como una "función atribuible a los docentes universitarios, quienes de forma transversal la ejercen y la enseñan como parte de los fines institucionales" (Parra-Moreno, 2002, p. 123). La investigación es un proceso sistemático de búsqueda y construcción del conocimiento, a partir de métodos rigurosos que permiten la validación de los saberes. De acuerdo a Hugo Cerda-Gutiérrez (2008), la investigación está íntimamente ligada al uso de técnicas de recolección y tratamiento de la información, pero ello supone la definición clara de un problema que se busca resolver. Por ello, Ciro Parra-Moreno (2002) indica que se debe estar alerta para que la investigación no sea reducida a un simple implante metodológico, cuyo rigor y validez se circunscriben a las técnicas e instrumentos utilizados; esto desconoce la verdadera finalidad del método, que es la solución de problemas particulares y concretos del hacer.

Las universidades son las instituciones reconocidas desde el punto de vista social, político y cultural para llevar a cabo los procesos de investigación (Maldonado, Landazábal, Hernández, Ruiz, Claro, Vanegas \& Cruz, 2007, p. 44; Miyahira-Arakaki, 2009, p. 119; Rojas-Betancur, 2009, p. 1598), tanto en su aplicación como en la enseñanza y, en consecuencia, los docentes cumplen un rol fundamental para la promoción de la investigación en diferentes escenarios. Carlos Augusto Hernández (2000) aclara que "la universidad tiene como tarea central apropiar y ampliar el saber necesario para el desarrollo colectivo" (p. 229). El estudiante y el docente deben valorar esencialmente su capacidad de prestar un servicio a la sociedad, y este objetivo debe ser mucho más fuerte y mucho más integrador que los intereses personales o de grupo. Cada día más, la sociedad requiere investigadores o expertos de diferentes áreas que se conviertan en docentes y socialicen todo ese bagaje académico.

Hugo Cerda-Gutiérrez (2007, p. 39) expone que la relación del maestro con la investigación se puede percibir en tres sentidos: a) el docente hace investigación; b) el docente aplica la investigación como una forma de docencia; y c) el docente integra los productos de investigación en su práctica y planeación. De acuerdo a lo anterior:

a. Desde su campo profesional, el docente debe plantear y desarrollar investigaciones innovadoras y pertinentes, que permitan la construcción del conocimiento y su transferencia. Esto significa que todo docente universitario participa en investigaciones formales y se convierte en docente investigador. Se concreta en la participación que tenga el docente en centros de investigaciones, grupos de investigación, publicaciones científicas, redes de investigación, desarrollo de proyectos institucionales, etc. (investigación productiva).

b. El docente utiliza la investigación como parte de su plan de estudios pues busca que sus estudiantes adquieran habilidades y competencias en investigación científica. Esto implica la combinación de su cátedra con la instrucción en metodología de la investigación, y en la enseñanza propia de la escritura académica basada en la revisión 
de la literatura, y la reflexión y discusión en torno a los resultados obtenidos en los antecedentes. En el desarrollo de la docencia, el profesor universitario demuestra con experiencias y de forma práctica las oportunidades y ventajas de conectar la profesión con la investigación vigente en cada campo (investigación formativa).

c. El docente universitario demuestra estar actualizado en las discusiones académicas y científicas, a partir del reconocimiento, la identificación, la sistematización y el análisis de la literatura. Esto le permite al docente transferir a los estudiantes los principales debates en torno a la ciencia o disciplina en que se inscribe la cátedra, además de los principales enfoques y métodos para el abordaje de los fenómenos que requieren ser estudiados (investigación formativa).

Por su parte, Luis Facundo Maldonado, Diana Patricia Landazábal, Juan Carlos Hernández, Yasbleidy Ruiz, Audrey Claro, Harvert Vanegas y Sandra Cruz (2007) especifican un conjunto de habilidades, actitudes y aptitudes propias de la competencia investigativa que se busca desarrollar entre los estudiantes de educación superior. Para el autor, la investigación formativa se centra en "las dimensiones epistemológica, metodológica, técnica y social” (p. 48), y ello se traduce en la formación de los estudiantes, para (1) describir propiedades de la acción investigativa, (2) categorizar y conceptualizar en función de interpretar la realidad a partir de teorías, modelos y enfoques, (3) identificar principios y normas que rigen la actividad investigativa y formativa; (4) reportar o socializar a la comunidad académica las ideas e interpretaciones producto de la acción investigativa, (5) se construyan formas de organización del quehacer investigativo, (6) formalicen modos de comunicación e interacción entre grupos de investigación o investigadores y la comunidad académica o productiva, promulgando la visibilidad de estas relaciones.

Para Bernardo Restrepo-Gómez (2002, p. 43), el problema de la relación entre la docencia y la investigación se observa desde la situación en que el investigador debe permitir el aprendizaje de la misma investigación y la transferencia del conocimiento producido. Para ello, el docente debe generar espacios y estrategias que permitan al estudiante la apropiación del conocimiento y el desarrollo de habilidades para la construcción del mismo, así como su transferencia y socialización. Según Bernard Honoré (1980, p. 50), la educación tiene como fin instaurar ambientes óptimos para que saberes procedentes del exterior puedan ser interiorizados, se acoplen internamente, consiguiendo así una transformación que pueda exteriorizar un nuevo saber enriquecido por los conocimientos del individuo que participa en la relación sujetoaprendizaje. Se trata de una propiedad acumulativa del conocimiento.

Desde el punto de vista de Martha Cecilia Gutiérrez-Giraldo (2005), se debe realizar de manera constante una propuesta para la formación de investigadores pues la cuestión prioritaria es la búsqueda de una pedagogía que facilite el proceso de enseñanza-aprendizaje. El objetivo de una pedagogía para la investigación formativa se concreta en la enseñanza de estrategias, procedimientos, enfoques y métodos 
propios de la investigación científica, el desarrollo de habilidades para buscar, sistematizar y hacer uso de la literatura, y la producción y análisis de textos académicos que permitan el fortalecimiento de una visión interdisciplinar y compleja. Jeremías Quiñones-Cárdenas y Consuelo Vélez-Álvarez (2004, p. 145) vislumbran que es necesario diversificar las instituciones educativas, para replantear el conjunto de operaciones, dispositivos y procedimientos que utilizan quienes desarrollan tareas encaminadas a la formación de nuevos investigadores, en busca de un verdadero proceso pedagógico, que geste un ser humano que indague entre las estrategias, los procedimientos, las nuevas formas de aprendizaje y la realización personal. En resumen, la formación investigativa en la universidad debe ser un eje de la pedagogía, y, por tanto, una apuesta para la construcción y recuperación de la actitud científica de los estudiantes (Rojas-Betancur, 2009, p. 1598).

Orlando Valera-Alfonso (1999) asume que los métodos de investigación pedagógica han traído como consecuencia la aplicación de una diversidad de tecnologías. Esto pone de manifiesto otro aspecto relevante de la investigación formativa: una pedagogía para la investigación debe facilitar el uso de las tecnologías de la informática y las comunicaciones, TIC, y se traduce en las posibilidades que generan los docentes con las prácticas pedagógicas para facilitar el uso y la apropiación de las TIC a fin de construir escenarios virtuales que promocionen la transferencia de los saberes. Al respecto, Ana García-Valcárcel Muñoz-Repiso (2007) señala:
El desarrollo tecnológico y las nuevas formas de comunicación obligan a la institución universitaria a replantearse la práctica educativa. Las tecnologías digitales de la información y la comunicación están teniendo un peso cada vez mayor en los procesos educativos universitarios, reclamando la configuración de nuevos espacios y ambientes de aprendizaje, así como nuevas funciones y roles profesionales en el profesorado (p. 126).

En el ámbito educativo, la forma de enseñar es muy importante y el punto de partida son las estructuras que reflejan los estudiantes para pensar y actuar. Para Myriam Zúñiga (1998), la práctica pedagógica como campo de reflexión crea una mayor exigencia para los docentes, ya que origina la necesidad de elaborar una práctica creativa apoyada en lecturas, prácticas sociales, investigaciones en el aula, producción de material y socialización de resultados de investigaciones. Se debe impulsar la liberación y la autonomía del individuo con el propósito de desarrollar su creatividad, capacidad innovadora y actitud crítica.

La investigación formativa se traduce en un problema pedagógico y didáctico, pues la cuestión central es cómo enseñar para promover un aprendizaje que facilite el desarrollo y potenciación de habilidades y competencias investigativas marcadas por la criticidad, interdisciplinariedad y flexibilidad (Rojas-Betancur, 2009). Así las cosas, la investigación formativa es un proceso pedagógico particular caracterizado por la interacción dinámica, reflexiva, transversal y multidireccional de los sujetos que en ella participan, es decir, docentes y 
LA INVESTIGACIÓN FORMATIVA EN LAS PRÁCTICAS DOCENTES / W. AVENDAÑo, G. RUEDA, L. PAZ / 165

estudiantes, y cuyo propósito es el aprendizaje efectivo de los elementos que integran las dimensiones epistemológica, metodológica, técnica y comunitaria de la investigación científica.

\section{Investigación formativa en los programas de contaduría pública de Colombia}

La contaduría como carrera universitaria busca formar contadores públicos competentes para realizar las actividades y desempeñar los cargos que la sociedad demanda en los siguientes campos de actuación: contable, auditoría y control, impuestos, financiero y administrativo. Los programas de estudio de contaduría pública en Colombia responden históricamente a la necesidad de formar profesionales según los requerimientos del mercado laboral (ArqueroMontaño, 2000; García-Jiménez \& BoteroGiraldo, 2014; Muñoz-López, Ruiz-Rojas \& Sarmiento-Ramírez, 2015; Murgueitio, 2011; Ordóñez; s.f.). Esta educación direccionada desde el mercado privilegia las acciones instrumentales que contribuyan con el conjunto eficiencia, eficacia, maximización, y en estos propósitos difícilmente se contempla posibilidad alguna para un contador reflexivo y crítico.

Para María Eugenia Upegui-Velásquez (2002), las facultades de contaduría pública en Colombia se orientan más hacia la enseñanza de la técnica, y dejan en un segundo plano el análisis de conceptos y el estudio de teorías. Una de las principales consecuencias de la ausencia de investigación en la disciplina contable es la falta de abordaje a conteni- dos conceptuales, de resolución de problemas y el pensamiento crítico, ya que se deja de lado por problemas de naturaleza estrictamente práctica, instrumentalista y utilitaria, pues se desarrolla un saber hacer, el cual no favorece procesos metacognitivos y poco se indaga sobre la naturaleza reflexiva de la situación propuesta y sus contenidos transdisciplinares (GarcíaJiménez \& Botero-Giraldo, 2014).

En los últimos años, ha quedado en evidencia la importancia de la investigación en el campo de formación profesional, surgiendo diversos estudios que indagan sobre su lugar en los programas de contaduría pública en el país. Según Ruth Alejandra Patiño-Jacinto y Gerardo Santos (2009), la investigación debe ser reconocida por el contador y las universidades formadoras de estos profesionales, como el medio de incorporación a la sociedad del conocimiento y como estrategia de generación de alternativas en una sociedad caracterizada por continuas transformaciones económicas, políticas y sociales.

Para Édgar Gracia-López (2010), actualmente las universidades contables transitan de currículos fragmentados a currículos integrados y problémicos en los que la investigación y sus resultados son vitales. Al respecto, Sandra Milena Muñoz-López, Gustavo Alberto Ruiz-Rojas y Héctor José Sarmiento-Ramírez (2015) plantean que si bien buena parte de los programas de contaduría pública en el país dejan entrever en sus estructuras curriculares algunas apuestas por la formación en otras disciplinas que dialogan con la contabilidad, aún no ha sido comprendida plenamente su potencialidad en la formación de contadores públicos. 
Es de sobra reconocido en el medio la escasa investigación contable y la poca incidencia que tiene en la formación del estudiante y en el devenir de la profesión, situación que le genera a la Contaduría Pública gran cantidad de inconvenientes, en tanto no logra interpretar correctamente su función social, ni ubicar ciertamente la solución a las problemáticas fundamentales (Gracia-López, 2002, p. 102).

Las dificultades en los procesos de investigación contable tienen su origen, en muchos de los casos, en el acto educativo cuando está desprovisto de referentes, contenidos y argumentaciones propios del área contable (Gracia-López, 2010, p. 267).

Para María Eugenia Upegui-Velásquez (2002), otra de las dificultades se relaciona con la escasa formación pedagógica de los profesores de estas universidades, que por sus condiciones personales y profesionales han sido vinculados al espacio educativo, sin ningún entrenamiento para cumplir a cabalidad esta labor.

En la enseñanza universitaria de contaduría pública, la investigación debe ser elemento constitutivo del currículo; esto es, "identificarse como un aspecto central y dinamizador de los criterios, planes de estudio, programas, metodologías, y procesos que contribuyen a la formación integral y a la construcción de la identidad cultural nacional, regional y local" (Patiño-Jacinto\& Santos, 2009, p. 24).

Roberto Antonio Ríos-León (2013), respecto al intento de inclusión parcial de procesos investigativos en el currículo, plantea que cuando los profesores desarrollan actividades de investigación, presentan en principio la teoría para luego llevar a cabo un ejercicio práctico en las organizaciones del entorno, el cual es presentado y sustentado por los estudiantes al finalizar el período académico, pero lo dejan sin continuidad ni difusión. Concluye que la importancia de la formación para la investigación está dada en que facilita tanto al profesor como al estudiante mantenerse vigentes y competitivos en temas de investigación, para la identificación de problemas, análisis de alternativas y propuestas de solución, al pasar de la teoría a la práctica y apropiarse del lenguaje propio del investigador.

Sergio Ordóñez (s.f.) plantea que gran parte de la investigación contable desarrollada en Colombia es desconocida por docentes y estudiantes, y que en aquellas universidades donde aparece la investigación se relega a los primeros semestres, con ausencia de planificación, y que es más bien un centro de coordinación y discusión sobre el trabajo de una serie de investigadores que se han vinculado pues cada uno ha desarrollado sus actividades de una manera autónoma e independiente y sus trabajos se orientan de acuerdo a los intereses particulares de cada miembro y no como respuesta a una tarea coordinada, estudiada y consensuada. Para el autor, los programas de estudio deben fomentar actividades académicas que permitan la discusión sobre temáticas inherentes a la profesión y disciplina contable. Estas discusiones deben ser articuladas a los planes de estudio de manera amplia, dejando de lado posiciones que toman las reflexiones epistemológicas, científicas o filosóficas como extrañas a la práctica contable. 
LA INVESTICACIÓN FORMATIVA EN LAS PRÁCtiCAS DOCENTES / W. AVENDAÑo, G. RUEDA, L. PAZ / 167

\section{Método}

El paradigma epistemológico de la investigación es empírico-analítico. Los paradigmas responden a la racionalidad científica dentro de la cual se inscribe la investigación que se realiza, y esto depende de la forma en que el investigador asume el fenómeno para su estudio y análisis más que en los métodos empleados (Fermoso, 1988-1989, p. 122).

En atención a los métodos empleados, se trata de una investigación en la que se aplicaron métodos cuantitativos a través de un diseño no experimental, es decir, un estudio en el que no hubo manipulación de las variables de análisis (Hernández-Sampieri, FernándezCollado \& Baptista-Lucio, 2008). En cuanto al tipo de investigación, se trata de un estudio descriptivo ya que tiene como objetivo indagar la incidencia de las modalidades o niveles de una o más variables en una población. De acuerdo a Mario Tamayo y Tamayo (2005), la investigación descriptiva tiene como propósito “la descripción, registro, análisis e interpretación de la naturaleza actual, y la composición o procesos de los fenómenos" (p. 35).

La población de la investigación está representada por sesenta (60) docentes adscritos al Departamento de Ciencias Contables y Financieras, que orientan como mínimo una asignatura del Programa de Contaduría Pública. En total, hay un docente con grado de doctor, ocho docentes con maestría, 31 docentes con grado de especialista y veinte docentes con nivel de pregrado. A la totalidad de la población se le aplicó el instrumento diseñado para la recolección de la información, el cual correspondió a un cuestionario estructurado (o encuesta) tipo Likert compuesto de 24 preguntas con opciones de respuesta siempre, casi siempre, a veces, casi nunca y nunca. El Instrumento para Medir Investigación Formativa y Práctica pedagógica (IMIFP) aplicado a la muestra descrita abordó diferentes variables relacionadas con la investigación formativa y las prácticas pedagógicas de los docentes a fin de encontrar las correlaciones entre variables que permitiesen interpretar el fenómeno.

La confiabilidad y la validez del instrumento resultaron fundamentales, pues como explica Carlos Eduardo Méndez-Álvarez (2001), "una medición es confiable o segura, cuando aplicada repetidamente a un mismo individuo o grupo, o al mismo tiempo por investigadores diferentes, obtiene resultados iguales o parecidos" (p. 222). La confiabilidad del instrumento fue determinada por el cálculo estadístico de fiabilidad alfa de Cronbach:

- La variable investigación formativa corresponde a las preguntas $1,2,3,4,5,6,13,14$, $15,16,17$ y 18 , con un valor de 0.842 .

- La variable de prácticas pedagógicas corresponde a las preguntas $7,8,9,10,11,12,19$, $20,21,22,23$ y 24 , con un valor de 0.863 .

Para la totalidad del instrumento - es decir, las 24 preguntas-, el análisis estadístico arrojó un valor de 0.910. Los anteriores resultados se interpretan como valores de confiabilidad altos, lo que asegura que la información recabada para cada una de las variables, y para el instrumento completo, es confiable y significativa.

En cuanto a la validez del instrumento —es decir, el "grado en que un instrumento 
realmente mide la variable que pretende medir" (Hernández-Sampieri, Fernández-Collado \& Baptista-Lucio, 2008, p. 236)—, se utilizó el juicio de expertos, que colaboraron en la evaluación metodológica y estadística del cuestionario, así como en la coherencia y redacción de cada uno de los ítems. De acuerdo a la validez por contenido, Ronald Jay Cohen, Mark Swerdlik y Edward Sturman (2001, p. 113) resaltan que esta es una prueba que establece un juicio que concierne al comportamiento de la representación de la población objeto de estudio, que es necesario cuantificar. Para ello se utilizó el método de Lawshe:

Razón de validez de contenido:

$$
R V C=\frac{n_{\varepsilon}-\left(\frac{N}{2}\right)}{\left(\frac{N}{2}\right)}
$$

A continuación, se presentan los resultados o hallazgos obtenidos con la aplicación del instrumento o encuesta a los 60 docentes del programa de contaduría públicade la Universidad Francisco de Paula Santander.

Donde:

$n_{\varepsilon}:$ Número de expertos que aprueban

$N$ : Número total de expertos

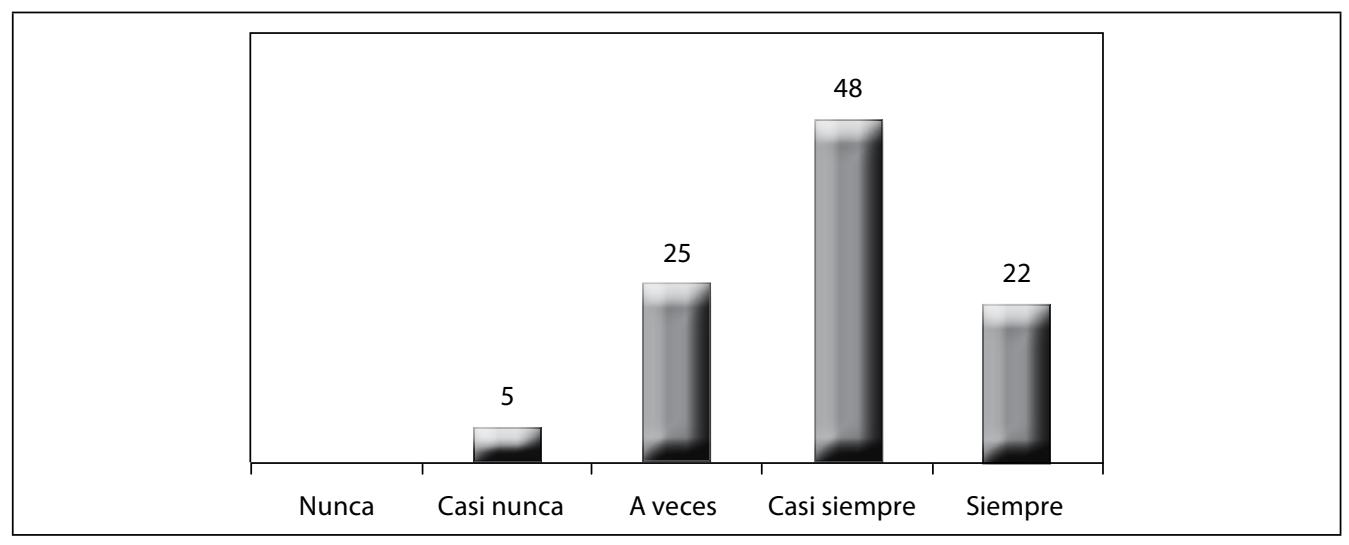

Gráfico 1

Revisión bibliográfica de los docentes como parte de su actividad pedagógica

Fuente: elaboración propia, a partir del SPSS 
La revisión bibliográfica es una de las actividades propias de la investigación formativa con mayor uso entre la población. El 70\% de los docentes describe que casi siempre o siempre in- dagan materiales científicos a fin de apoyar sus actividades pedagógicas, profundizar en conocimientos específicos y mantener una actualización permanente en el campo del saber.

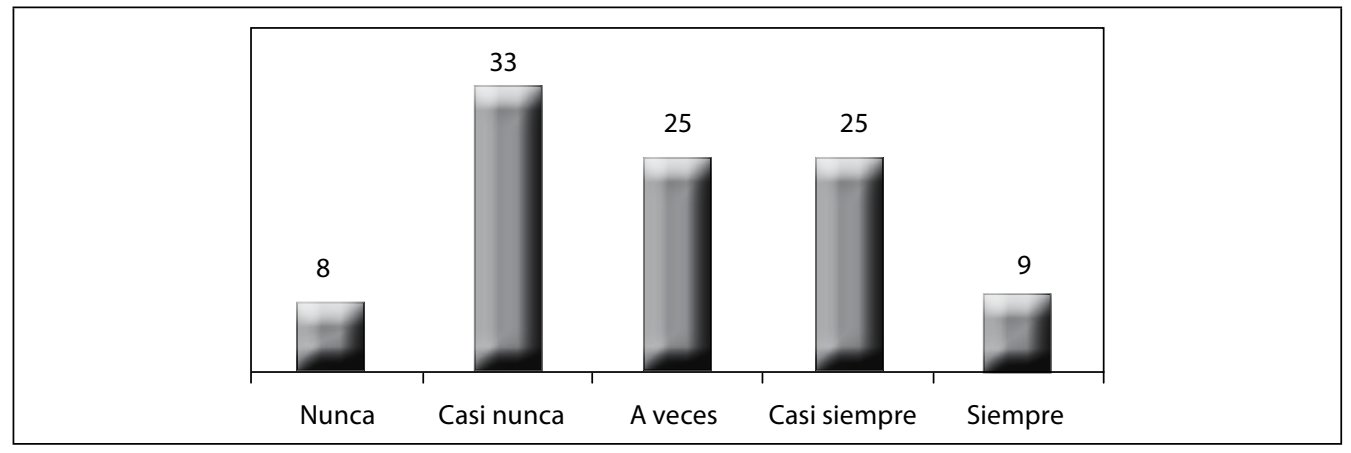

\section{Gráfico 2}

Participación activa en la investigación a fin de producir saberes (investigación productiva) Fuente: elaboración propia, a partir del SPSS

La respuesta de los docentes encuestados evidencia su poca participación en actividades formales de investigación productiva y desarrollo de nuevos saberes. Esto refleja el bajo nivel de relación que tienen los docentes del programa académico con algunas exigencias para las universidades ante la denominada sociedad del conocimiento. Por lo general, no participan en proyectos de investigación y producción científica, no se observó financiación de proyectos por instituciones o entidades, y muy pocos grupos de investigación mantienen una activa participación en el campo científico.

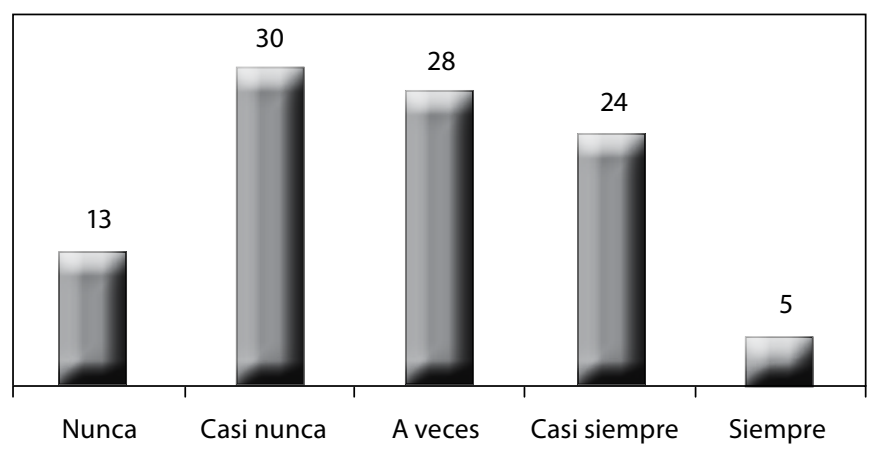

\section{Gráfico 3}

Desarrollo de investigaciones con apoyo de los estudiantes Fuente: elaboración propia, a partir del SPSS 
La casi totalidad de las respuestas obtenidas muestra que los docentes no realizan investigaciones acompañados por los estudiantes. Respecto a aquellos docentes que sí reflejan la investigación en conjunto con estudiantes, se destacan valores similares al gráfico anterior, respecto a quienes realizan investigaciones.

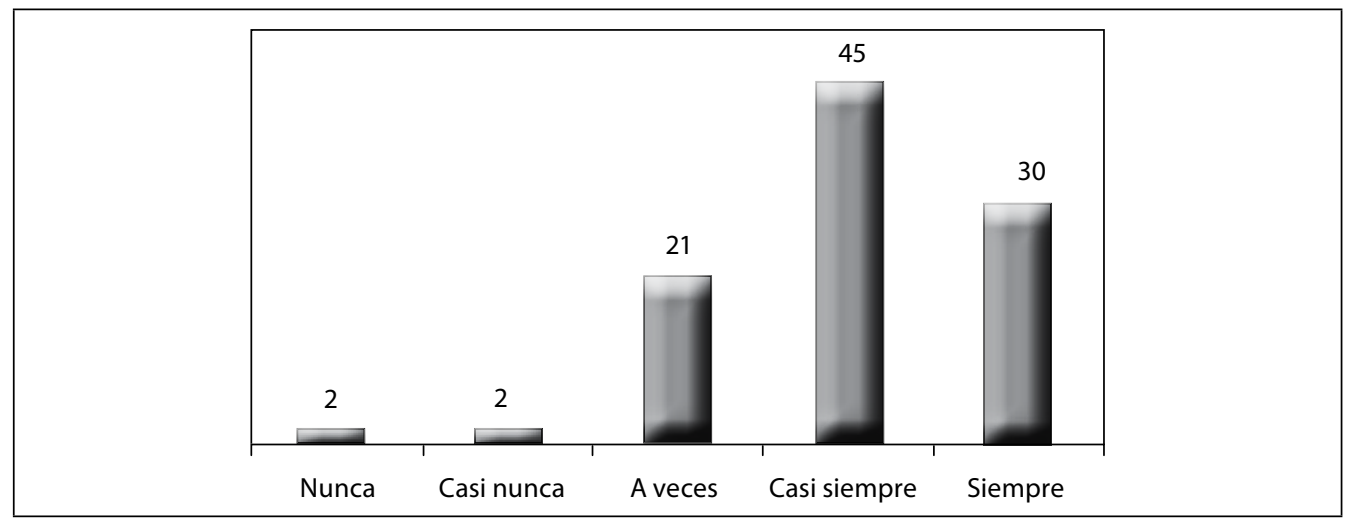

\section{Gráfico 4}

Reflexión de la praxis pedagógica y evaluación del desempeño por parte de los docentes Fuente: elaboración propia, a partir del SPSS

Un porcentaje alto de los docentes encuestados reflexionan sobre su actividad y praxis pedagógica, y evalúan su desempeño frente a la asignatura. Esto significa que los docentes intentan manejar un componente pedagógico que les permita internalizar aquellos saberes que transforman su actividad, y realizan un examen personal respecto a lo que generan con esa transformación interna del saber.

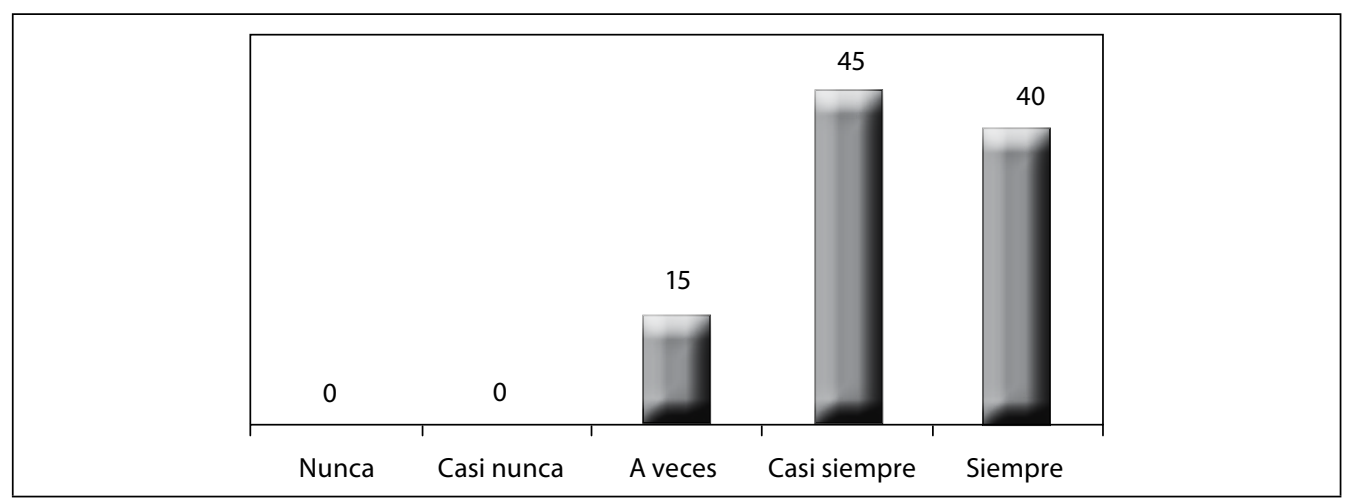

Gráfico 5

Posición crítica frente a la información a emplear en las prácticas pedagógicas

Fuente: elaboración propia, a partir del SPSS 
De los 60 encuestados, la gran mayoría sostiene que tienen una posición crítica respecto de la información que emplean en el desarrollo de las asignaturas.

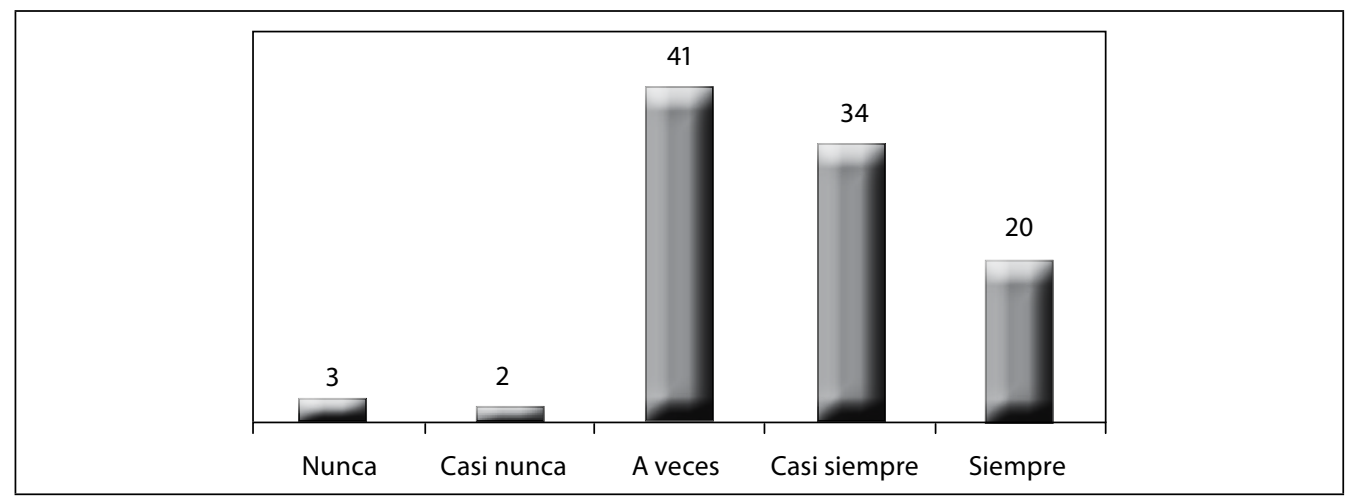

Gráfico 6

Desarrollo de foros de discusión para dar sentido a la información Fuente: elaboración propia, a partir del SPSS

Al observar la respuesta obtenida, la mayoría de los docentes crean y desarrollan espacios de diálogo con sus estudiantes para dar sentido a las lecturas o revisiones bibliográficas que se llevan a cabo dentro de la asignatura. Esta actividad, si bien no es la única para la socialización de los saberes, representa la estrategia con mayor utilidad académica. Los foros de discusión permiten aclarar dudas, exponer puntos de ten- sión epistemológica y metodológica, reconocer la diversidad de saberes y llegar a consensos que permitan ubicar los enfoques de interpretación.

A continuación, se presenta el análisis de datos correspondiente a las correlaciones de variables para confrontar la investigación formativa y las prácticas docentes empleadas por los individuos a los que se les aplicó el instrumento.

\begin{tabular}{llcccc}
\hline & Valor & $\begin{array}{c}\text { Error típ. } \\
\text { asint. }^{\text {a }}\end{array}$ & $\begin{array}{c}\text { T. } \\
\text { aproximada }^{b}\end{array}$ & $\begin{array}{c}\text { Sig. } \\
\text { aproximada }^{*}\end{array}$ \\
\hline \multirow{3}{*}{ Ordinal por ordinal } & Tau-b de Kendall &, 301 &, 125 & 2,389 &, 017 \\
& Tau-c de Kendall &, 264 &, 110 & 2,389 &, 017 \\
& Gamma &, 425 &, 170 & 2,389 &, 017 \\
Medida de acuerdo & Kappa &, 270 &, 090 & 3,188 &, 001 \\
\hline
\end{tabular}

Tabla 1

Correlación de variables de los ítems 1 y 7 (trabajos de revisión bibliográfica y reflexión de la práctica del desempeño docente para el desarrollo de planes de mejoramiento)

a Asumiendo la hipótesis alternativa

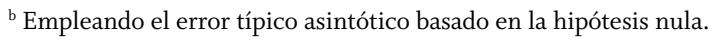

Fuente: elaboración propia 
La tabla 1 refleja una correlación con un valor $\mathrm{p}<0.05$, lo que se traduce en una correlación significativa mayor a 95\%. Esto indica que la revisión bibliográfica realizada por los docentes corresponde a una actividad que promueve el proceso de reflexión y evaluación pedagógica, es decir, a medida que se explora la literatura sobre el campo de saber específico, los docentes definen criterios para su reflexión pedagógica y práctica docente y disciplinar. El resultado de esta actividad se concreta en acciones y planes de mejoramiento sobre los contenidos programáticos de las asignaturas y el diseño de nuevas experiencias significativas que promuevan capacidades, competencias y habilidades entre los estudiantes.

\begin{tabular}{llcccc}
\hline & Valor & $\begin{array}{r}\text { Error típ. } \\
\text { asint. }^{\text {a }}\end{array}$ & T. aproximada $^{b}$ & $\begin{array}{c}\text { Sig. } \\
\text { Aproximada }\end{array}$ \\
\hline \multirow{3}{*}{ Ordinal por ordinal } & Tau-b de Kendall &, 295 &, 111 & 2,601 &, 009 \\
& Tau-c de Kendall &, 258 &, 099 & 2,601 &, 009 \\
& Gamma &, 406 &, 147 & 2,601 &, 009 \\
Medida de acuerdo & Kappa &, 130 &, 070 & 2,178 &, 029 \\
\hline
\end{tabular}

Tabla 2

Correlación de variables de los ítems 2 y 9 (compartir con estudiantes experiencias de investigación y contextualización de los ejercicios prácticos con investigaciones propias)

${ }^{\text {a }}$ Asumiendo la hipótesis alternativa

${ }^{\mathrm{b}}$ Empleando el error típico asintótico basado en la hipótesis nula. Fuente: elaboración propia

La tabla 2 refleja una correlación con un valor $\mathrm{p}<0.05$, lo que se traduce en correlación significativa mayor de 95\%. Dicho de otra manera, los docentes que comparten experiencias de investigación propias con los estudiantes en el desarrollo de las asignaturas promueven la contextualización del conocimiento y de los saberes propios de la disciplina. La investigación productiva de los docentes les permite mejorar las prácticas pedagógicas y, en especial, la investigación formativa pues transfiere entre los sujetos de formación aspectos epistemológicos, teóricos y metodológicos de utilidad para ellos. Debido a que muchos docentes que participaron en la investigación no desarrollan actividades propias de la investigación productiva, los resultados se reflejan en una baja capacidad de ellos para ejercer una investigación formativa eficaz.

\begin{tabular}{llcccc}
\hline & Valor & $\begin{array}{c}\text { Error típ. } \\
\text { asint. }^{\text {a }}\end{array}$ & T. aproximadab $^{\text {a }}$ & $\begin{array}{c}\text { Sig. } \\
\text { aproximada }\end{array}$ \\
\hline \multirow{3}{*}{ Ordinal por ordinal } & Tau-b de Kendall &, 056 &, 114 &, 489 &, 625 \\
& Tau-c de Kendall &, 056 &, 114 &, 489 &, 625 \\
& Gamma &, 082 &, 168 &, 489 &, 625 \\
Medida de acuerdo & Kappa &, 049 &, 077 &, 685 &, 494 \\
\hline
\end{tabular}

Tabla 3

Correlación de variables de los ítems 3 y 8 (considerar las experiencias de aula de otros compañeros docentes y posición crítica frente a la información utilizada en el aula)

${ }^{a}$ Asumiendo la hipótesis alternativa

${ }^{\mathrm{b}}$ Empleando el error típico asintótico basado en la hipótesis nula.

Fuente: elaboración propia 
La tabla 3 refleja una correlación con un valor $\mathrm{p}>0.05$, expresando así que es una correlación no significativa. En otros términos, se observa que si bien los docentes analizan la información que emplean en el desarrollo de sus asignaturas, no reflexionan sobre las prácticas que hacen sus compañeros docentes del programa.

\begin{tabular}{llcccc}
\hline & Valor & $\begin{array}{c}\text { Error típ. } \\
\text { asint. }^{\text {a }}\end{array}$ & T. aproximada $^{\text {b }}$ & $\begin{array}{c}\text { Sig. } \\
\text { aproximada }\end{array}$ \\
\hline \multirow{3}{*}{ Ordinal por ordinal } & Tau-b de Kendall &, 042 &, 127 &, 329 &, 742 \\
& Tau-c de Kendall &, 038 &, 116 &, 329 &, 742 \\
& Gamma &, 055 &, 166 &, 329 &, 742 \\
Medida de acuerdo & Kappa &, 129 &, 081 & 1,831 &, 067 \\
\hline
\end{tabular}

Tabla 4

Correlación de variables de los ítems 4 y 9 (autoconcepto de capacidad para el desarrollo de investigaciones y contextualización de los ejercicios prácticos con investigaciones propias)

${ }^{a}$ Asumiendo la hipótesis alternativa

${ }^{\mathrm{b}}$ Empleando el error típico asintótico basado en la hipótesis nula.

Fuente: elaboración propia

La tabla 4refleja una correlación con un valor $\mathrm{p}>0.05$, lo que expresa que es una correlación no significativa. Esto se traduce en que los docentes que participaron en el estudio en su mayoría consideran que tienen las capacidades y cualidades requeridas para desarrollar distintas formas de investigación, pero en esta tarea de investigación productiva no abordan o consideran aquellos estudios o ideas de investigación que tienen su origen dentro del aula.

\begin{tabular}{llcccc}
\hline & Valor & $\begin{array}{c}\text { Error típ. } \\
\text { asint. }^{\text {a }}\end{array}$ & $\begin{array}{c}\text { T. } \\
\text { aproximada }^{\text {b }}\end{array}$ & $\begin{array}{c}\text { Sig. } \\
\text { aproximada }\end{array}$ \\
\hline \multirow{3}{*}{ Ordinal por ordinal } & Tau-b de Kendall &, 324 &, 116 & 2,772 &, 006 \\
& Tau-c de Kendall &, 306 &, 110 & 2,772 &, 006 \\
& Gamma &, 409 &, 142 & 2,772 &, 006 \\
Medida de acuerdo & Kappa &, 095 &, 082 & 1,439 &, 150 \\
\hline
\end{tabular}

Tabla 5

Correlación de variables de los ítems 5 y 10 (adopción de otras investigaciones pedagógicas desarrolladas para aplicar en el aula y enseñar como extraer y organizar la información disponible)

${ }^{\text {a }}$ Asumiendo la hipótesis alternativa

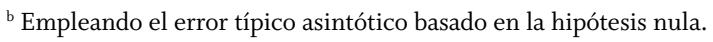

Fuente: elaboración propia

La tabla 5 muestra una correlación con un valor $\mathrm{p}<0.05$, lo que se traduce en correlación significativa mayor de 95\%. Esto significa que los docentes que participaron en el estudio en ocasiones hacen revisiones sobre investigaciones de naturaleza pedagógica con el fin de aplicar metodologías, intervenciones o modelos que han sido puestos en práctica en otros es- 
cenarios, con el propósito de reproducir prácticas de enseñanza-aprendizaje que faciliten la transferencia de la información entre los estudiantes.

\begin{tabular}{llcccc}
\hline & Valor & $\begin{array}{c}\text { Error } \\
\text { típ. } \\
\text { asint. }^{\text {a }}\end{array}$ & T. aproximada $^{\text {b }}$ & $\begin{array}{c}\text { Sig. } \\
\text { aproximada }\end{array}$ \\
\hline \multirow{3}{*}{ Ordinal por ordinal } & Tau-b de Kendall &, 242 &, 125 & 1,901 &, 057 \\
& Tau-c de Kendall &, 222 &, 117 & 1,901 &, 057 \\
& Gamma &, 318 &, 159 & 1,901 &, 057 \\
Medida de acuerdo & Kappa &, 209 &, 081 & 3,102 &, 002 \\
\hline
\end{tabular}

Tabla 6

Correlación de variables de los ítems 6 y 11 (uso de métodos para el registro de estrategias educativas promovidas en el aula y facilitar herramientas a los estudiantes para la elaboración de textos escritos)

a Asumiendo la hipótesis alternativa

${ }^{\mathrm{b}}$ Empleando el error típico asintótico basado en la hipótesis nula.

Fuente: elaboración propia

La tabla 6 muestra una correlación con un valor $\mathrm{p}>0.05$, lo que expresa que es una correlación no significativa. Esto representa que si bien los docentes buscan facilitar que los estudiantes desarrollen habilidades que favorezcan la escritura académica mediante la exploración de diversos tipos de escritos (ensayos, artículos, protocolos, textos críticos, etc.), no tienen en cuenta los métodos de registro que permitan aumentar los saberes disciplinares.

\begin{tabular}{ccc}
\hline Relaciones significativas & Relaciones NO significativas & Total correlación por ítem \\
\hline 7 & 5 & 12 \\
7 & 5 & 12 \\
7 & 5 & 12 \\
5 & 7 & 12 \\
6 & 6 & 12 \\
5 & 7 & 12 \\
1 & 11 & 12 \\
4 & 8 & 12 \\
11 & 1 & 12 \\
7 & 5 & 12 \\
6 & 6 & 12 \\
9 & 3 & 12 \\
75 & 69 & 144 \\
\hline
\end{tabular}

Tabla 7

Correlación general de las variables

Fuente: elaboración propia 
Un análisis general de las correlaciones permitió evidenciar que las actividades de investigación están relacionadas con las prácticas pedagógicas, dando como resultado general un mayor número de relaciones de variables significativas, esto indicaría que aunque no se realiza investigación en sentido estricto, los docentes ponen en práctica la formación en investigación en su quehacer diario y la imparten con el grupo de estudiantes que tienen bajo su responsabilidad. No obstante, cuando se analizan los resultados por separado, la percepción que se obtiene es un escaso trabajo en investigación formativa.

\section{Discusión}

Se debe resaltar que los docentes realizan revisiones bibliográficas y que de forma constante renuevan la forma de transmitir los conocimientos, lo que incluye tener una posición clara con la información obtenida por ellos y sus estudiantes en el desarrollo de las asignaturas. Por otra parte, los docentes deberían tener una mayor preocupación por generar un plan para hacer seguimiento a los proyectos que se inician en el aula y que poseen un contenido profesional, de tal manera que haya un esfuerzo por culminar aquellos que los estudiantes generan bajo la guía del docente a cargo de la asignatura. Esto es un asunto primordial porque estas prácticas permiten enlazar la investigación formativa con la investigación productiva $y$, en consecuencia, promover el interés de los estudiantes que encontrarán que sus ideas de investigación en el aula pueden ser llevadas a campos más formales de carácter científico.
Además, esto respondería al modo en que se desarrolla la investigación formativa y la necesidad de un direccionamiento estratégico que promocione la investigación, que se promulga como exigencia formal a las universidades de Colombia, pues el Decreto 1295 de 2010 (Colombia, 2010) establece que se deben diseñar e implementar actividades de investigación que les permitan a los estudiantes adquirir una actitud crítica y una capacidad innovadora en torno a las ciencias, disciplinas, tecnologías, artes y/o humanidades.

Si bien se ha dado una distinción entre investigación formativa y productiva en cuanto a características, funciones y propósitos, estos dos tipos de investigación resultan interdependientes y complementarios. En otras palabras, el fortalecimiento de la investigación formativa depende en gran parte de la participación activa de los docentes en los grupos de investigación. Es posible que los bajos niveles de investigación productiva en la universidad se deban a la poca incorporación de los docentes en los grupos de investigación y en los proyectos institucionales que se gestionan. A su vez, esta limitación repercute en las aulas y en la investigación formativa pues los docentes en su mayoría - al no participar en la investigación científica formal- no actualizan sus conocimientos y prácticas, que se convierten en conductas rutinarias de enseñanza-aprendizaje.

Lo anterior se relaciona con los resultados obtenidos pues el 29\% de los docentes participa de forma activa en la investigación productiva y el 34\% busca que los estudiantes participen en investigaciones formales más allá de las aulas. A su vez, se presenta una correlación de estos 
resultados con el nivel académico de los docentes, pues aquellos con posgrados de más alto nivel son los docentes con mayor capacidad para ejercer prácticas de investigación formativa y productiva, y crear puentes entre la una y la otra. Del mismo modo, estos datos se corresponden con otros resultados que indican que los estudiantes son poco incorporados a procesos de investigación formales, lo que limita su potencial y colaboración en estas dinámicas significativas.

A pesar de que los docentes hacen una reflexión permanente sobre sus prácticas pedagógicas, los hallazgos demuestran que esta capacidad de autoevaluación no genera resultados concretos o cambios significativos hacia la mejora de la investigación formativa. Esto significa que no basta con la reflexión interna en torno a las prácticas pedagógicas que se realizan, pues esta actividad debe ser exteriorizada, socializada y concretada en acciones específicas que impacten la relación enseñanza-aprendizaje.

Con relación a la pedagogía, Myriam Zúñiga (1998) sostiene que el docente debe reflexionar para elaborar una práctica creativa apoyada en lecturas, prácticas sociales, investigaciones en el aula, producción de material, socialización de resultados de investigaciones realizadas por el docente, entre otras actividades, con el propósito de mejorar el nivel educativo y las capacidades de investigación. Por otra parte, Francisco Gutiérrez (1997) indica que en la constante búsqueda de una propuesta para la formación de investigadores se debe partir de una anotación previa que no busca la formación de investigadores en el campo de la pedagogía, sino que la preocupación es hacer que la formación de investigadores sea pedagógica, para desarrollar desde la práctica pedagógica aquellas capacidades que hagan del investigador un ser humano con buenas relaciones personales.

Conforme a los resultados, si bien se destacan varias prácticas válidas y significativas hacia la investigación formativa, aún se requiere fortalecer otras que son indispensables para mejorar los resultados. En la realidad, es evidente que los estudiantes en su gran mayoría sienten apatía por las actividades de investigación científica, siendo un llamado a todos los actores universitarios (docentes y directivos) para que aúnen esfuerzos. Y esto puede estar relacionado con el bajo impacto de las acciones pedagógicas diseñadas e implementadas por los docentes hacia la investigación formativa, y a los escasos recursos dispuestos por la Universidad para el desarrollo de investigaciones formales.

El docente debe generar espacios y estrategias que permitan al estudiante la apropiación del nuevo conocimiento y denominar este acto como aprendizaje por descubrimiento. Es necesario establecer estrategias de enseñanza, que canalicen los esfuerzos para obtener una transformación del conocimiento como individuo. Por tanto, no basta con los foros de discusión que fueron identificados en este estudio, pues la investigación formativa se enriquece con una gran variedad de prácticas. Antonio Latorre-Beltrán y Ramona González (1992, p. 31) afirman que con el surgimiento de nuevas prácticas, se da origen a una generación de maestros que tienen una óptica más reciente en la relación docente-estudiante, situación que se refleja de manera directa en la enseñanza- 
aprendizaje, la cual se centra en el estudiante para convertirlo en un sujeto activo de esta relación educativa. Esto mismo sucede con la investigación formativa o, aún más, esta puede servir de base para fortalecer el denominado aprendizaje por descubrimiento.

Es necesario aclarar - según lo planteado por Carlos Augusto Hernández (2000, p. 229) - que la universidad tiene como tarea central apropiar y ampliar el saber necesario para el desarrollo colectivo, en el cual el estudiante, el maestro y el maestro-investigador deben valorar esencialmente su capacidad de prestar un servicio a la sociedad. Este objetivo debe ser mucho más fuerte, mucho más integrador que los intereses personales o de grupo y las distintas voluntades encontradas en la institución con el propósito de transferir saberes que se conviertan en el insumo de las diferentes asignaturas. En la actualidad, existe la necesidad de convertir al investigador en docente pues esto configura una exigencia de la sociedad del conocimiento. Su reconocimiento genera confianza en los estudiantes, debido a que se refleja el dominio que posee el profesor a cargo de la asignatura de los conocimientos disciplinares requeridos en la carrera.

Para todo docente se hace necesario conocer cómo interactúan los estudiantes frente a los objetivos a alcanzar al finalizar la asignatura. De acuerdo a Bernardo Restrepo-Gómez (1997, p. 25), los conceptos de la investigación educativa enfatizan en realizar estudios históricos de la pedagogía, que analizan cómo se ha generado el espacio intelectual necesario para el mejoramiento de la educación e investigan el desarrollo del acto de enseñanza y todos los fac- tores que lo rodean. Renée Ávila (2005, p. 75), en cuanto a la investigación educativa, destaca que hay nuevas tendencias de carácter fenomenológico crítico, las cuales coinciden en la necesidad del retorno del sujeto y la revitalización de lo humano, situaciones que deben estar presentes en los procesos sociales y educativos abordados en el marco de una perspectiva histórica, cultural y social, cuyo centro de interés sea la persona, su vida cotidiana, sus creencias, simbolismos y cultura en general.

En el desarrollo de la actividad docente, es necesario realizar indagaciones en el aula sobre el modelo de enseñanza que se está aplicando y su trayectoria pedagógica, pues esto permite obtener un conocimiento de los factores que inciden en el acto educativo. En efecto, la mayor preocupación de los docentes en cuanto a su quehacer educativo, por lo general, es culminar el contenido programático de la asignatura y pasan por alto la adaptación que tienen los estudiantes frente a los objetivos propuestos en cada área del saber. Por lo anterior, también se requiere que la investigación productiva se centre en el acto educativo y en temas tan esenciales como los procesos de enseñanzaaprendizaje, la formación en pedagogía, la didáctica, el acto comunicativo en el marco de la educación, el desarrollo cognitivo y otros tantos ámbitos del conocimiento que deben ser objeto de la investigación educativa.

\section{Consideraciones finales}

A partir de la investigación realizada, se infiere que los sujetos objeto de análisis poco se involucran en prácticas alternativas e innovadoras 
relacionadas con la investigación formativa entendidas como el conjunto de acciones orientadas a promover la apropiación y el desarrollo de los conocimientos, las habilidades y las actitudes necesarios para llevar adelante una investigación científica.

Se considera que uno de los factores causales de esta problemática es el bajo nivel de investigación productiva entre los docentes, que poco participan en los grupos de investigación, el desarrollo de proyectos o en semilleros de investigación. Se hace necesaria una mayor vinculación de los docentes con la investigación productiva y la socialización de prácticas docentes significativas a fin de actualizar permanentemente sus concepciones frente al saber contable y sus concepciones frente a la profesión, transferir nuevos saberes al aula y potenciar los resultados en el espectro de la investigación formativa.

Aunque los docentes manifiestan mantener una posición crítica frente a la información, la realidad es que la ausencia de prácticas de investigación productiva permite inferir que esta variable debe ser analizada con mayor detenimiento. La investigación formativa depende en gran parte de las capacidades, habilidades y conocimientos que posee el docente en su campo epistemológico, y estos a su vez son desarrollados con la ejecución de prácticas en la investigación formal. Esto significa que los docentes cuyas prácticas tienen un impacto significativo en la investigación formativa son aquellos que se adentran en el conocimiento, y esto no se logra solo con revisiones bibliográficas. Se aprecia nuevamente la necesidad de capacitar al profesor universitario en aspectos pedagógicos, para lograr que sus alumnos reciban el conocimiento necesario y adquieran las habilidades para transformarlo o crearlo.

A fin de fortalecer la investigación formativa en el programa de contaduría pública, resulta perentorio que aquellas ideas de investigación o proyectos surgidos en el aula puedan trascender de este espacio con el propósito de fortalecer tanto la investigación formativa como la productiva. La investigación formativa debería permear el currículo de estudios.

Es posible que el nivel académico de los docentes influya en el bajo nivel de la investigación formativa, pues de los 60 docentes que participaron en el estudio, tan solo nueve cuentan con estudios de maestría y doctorado. Por tanto, la Universidad debe aunar esfuerzos para promover no solo los grupos de investigación, los semilleros o la producción académica de los docentes, sino que además está obligada a brindar oportunidades para el desarrollo de estudios de posgrado en los más altos niveles a todo su cuerpo de profesores. Se debe reconocer la importancia en la formación de los profesionales docentes.

Se hace imperiosa una revisión de la didáctica de la enseñanza de la investigación contable, que abra el debate sobre los conceptos centrales que se ponen en juego en los procesos de formación investigativa, la forma de presentación de ellos y los objetivos perseguidos.

\section{Referencias}

Arquero-Montaño, José Luis (2000). Capacidades no técnicas en el perfil profesional en contabilidad: las opiniones de docentes y profesionales. Revista Española de Fi- 
nanciación y Contabilidad, 29 (3), 149-172.

Disponible en: http://aeca.es/old/refc_1972-

2013/2000/103-6.pdf

Ávila, Renée (2005). Investigación educativa y cambio social. Bogotá: Ágora.

Bain, Ken (2004). What the Best College Teacher Do. Boston: Harvard University Press.

Bello, María Eugenia (2003).Educación y globalización: los discursos educativos en Iberoamérica. Barcelona: Anthropos.

Bocanegra-Acosta, Henry (2006). La investigación formativa: propuestas y retos en la formación de juristas.

Diálogos de Saberes, 24, 7-10. Disponible en: https://dialnet.unirioja.es/descarga/ articulo/2740963.pdf

Cerda-Gutiérrez, Hugo (2007). La investigación formativa en el aula. Bogotá: Magisterio.

Cerda-Gutiérrez, Hugo (2008). Los elementos de la investigación: cómo reconocerlos, diseñarlos y construirlos. Bogotá: Búho.

Cohen, Ronald Jay; Swerdlik, Mark \& Sturman, Edward (2001). Psychological Testing and Assessment: An Introduction to Tests and Measurement. Nueva York: McGraw-Hill.

Colombia (2010). Decreto 1295, 20 de abril de 2010. Diario Oficial, 47.687, 21 de abril de 2010. Disponible en: http://www. alcaldiabogota.gov.co/sisjur/normas/ Norma1.jsp?i=39363

Fermoso, Paciano (1988-1989). El modelo fenomenológico de investigación en pedagogía social. Educar, 14-15, 121-136. Disponible en: http://www.raco.cat/index. php/educar/article/viewFile/42218/90165 García-Jiménez, Marlon D. \& Botero-Giraldo, Gloria P. (2014). Entre la vida académica y la práctica profesional: problemas en la formación de los contables colombianos. Contexto, 3, 174-179. Disponible en: http:// revistas.ugca.edu.co/index.php/contexto/ article/download/287/597

García-Valcárcel Muñoz-Repiso, Ana (2007). Herramientas tecnológicas para mejorar la docencia universitaria. Una reflexión desde la experiencia y la investigación. Revista Iberoamericana de Educación a Distancia, RIED, 10 (2), 125-148. Disponible en: http://revistas.uned.es/index.php/ried/ article/viewFile/996/913

Gracia-López, Édgar (2002). Estado actual de la educación contable en Colombia. En Olver Quijano-Valencia, Édgar Gracia-López, Guillermo Martínez-Pino, Danilo Ariza-Buenaventura \& William Rojas-Rojas. Del hacer al saber: realidades y perspectivas de la educación contable en Colombia, 85-111. Popayán: Universidad del Cauca.

Gracia-López, Édgar (2010). La investigación contable en el acto educativo. Contaduría Universidad de Antioquia, 57, 255-269. Disponible en: http://aprendeenlinea.udea. edu.co/revistas/index.php/cont/article/ view/15588/13530

Guerrero-Useda, María Eugenia (2007). Formación de habilidades para la investigación desde el pregrado. Acta Colombiana de Psicología, 10 (2), 190-192. Disponible en: http://www.scielo.org.co/ pdf/acp/v10n2/v10n2a18.pdf Gutiérrez, Francisco (1997). Hacia una propuesta alternativa para la formación de investigadores. Nómadas, 7, 87-95. Disponible en: https://www.ucentral. 
edu.co/images/editorial/nomadas/docs/ nomadas_7_7_hacia_una_propuestas. pdf

Gutiérrez, Marina Yolanda \& Langarica, Guadalupe (1994). El perfil del docente de tiempo completo en la ENP [Escuela Nacional Preparatoria]. Perfiles Educativos, 64, 46-58. Disponible en: http://www. iisue.unam.mx/perfiles/perfiles_articulo. php?clave=1994-64-46-58

Gutiérrez-Giraldo, Martha Cecilia (2005). La formación pedagógica investigativa en estudiantes de formación avanzada en educación. Revista de Ciencias Humanas, 35 (1), 173-181. Disponible en: http://revistas. utp. edu.co/index.php/chumanas/article/ viewFile/1073/587

Hernández, Carlos Augusto (2000). La universidad y la vigencia de la cultura académica. Nómadas, 12, 225-232. Disponible en: http://www.redalyc.org/ pdf/1051/105115263021.pdf

Hernández-Sampieri, Roberto; FernándezCollado, Carlos\& Baptista-Lucio, Pilar (2008). Metodología de la investigación. México: McGraw-Hill. Disponible en: https://www.esup. edu.pe/descargas/dep_ investigacion/Metodologia\%20de $\% 20$ la\%20investigaci\%C3\%B3n\%205ta\%20 Edici\%C3\%B3n.pdf

Hewitt-Dundas, Nola (2012). Research Intensity and Knowledge Transfer Activity in UK Universities. Research Policy, 41 (2), 262-275.

Honoré, Bernard (1980). Para una teoría de la formación: dinámica de la formatividad. Madrid: Editorial Narcea.
Hurtado de Barrera, Jacqueline (2002). Formación de investigadores. Retos y alternativas. Bogotá: Magisterio.

Jones, Benjamin F.; Wuchty, Stefan \& Uzzi, Brian (2008). Multi-University Research Teams: Shifting Impact, Geography, and Stratification in Science. Science, 322 (5905), 1259-1262. Disponible en: http:// www.kellogg.northwestern.edu/faculty/ uzzi/ftp/081121\%20Multiuniversity\%20 teams\%20Science\%20WJ.and\%20Uzzi.pdf

Latorre-Beltrán, Antonio \& González, Ramona (1992). El maestro investigador. La investigación en el aula. Barcelona: Graó.

Maldonado, Luis Facundo; Landazábal, Diana Patricia; Hernández, Juan Carlos; Ruiz, Yasbleidy; Claro, Audrey; Vanegas, Harvert \& Cruz, Sandra (2007). Visibilidad y formación en investigación. Estrategias para el desarrollo de competencias investigativas. Revista Studiositas, 2 (2), 43-56. Disponible en: http://datateca. unad.edu.co/contenidos/301501/H_ MALDONADO_LANDAZABAL1.pdf Méndez-Álvarez, Carlos Eduardo (2001). Metodología. Diseño y desarrollo del proceso de investigación con énfasis en ciencias empresariales. Bogotá: McGraw Hill.

Miyahira-Arakaki, Juan M. (2009). La investigación formativa y la formación para la investigación en el pregrado. Revista Médica Herediana, 20 (3), 119-122. Disponible en: http://www.upch.edu.pe/ vrinve/dugic/revistas/index.php/RMH/ article/view/1010/976

Muñoz-López, Sandra Milena; Ruiz-Rojas, Gustavo Alberto \& Sarmiento-Ramírez, 
Héctor José (2015). Didácticas para la formación en investigación contable: una discusión crítica de las prácticas de enseñanza. Revista Facultad de Ciencias Económicas: Investigación y Reflexión, 23 (1), 53-86. Disponible en: http://www.redalyc.org/ pdf/909/90933063004.pdf

Murgueitio, Magdalida (2011). La investigación formativa como eje dinamizador hacia un pensamiento crítico del ejercicio contable. Gestión y Desarrollo, 8 (1), 79-94. Disponible en: http://www.usbcali.edu.co/sites/default/ files/3_investigacionformativa.pdf

Ordóñez, Sergio (s.f.). ¿Contribución de la investigación contable a la formación del contador público en Colombia? Cali, Colombia: Universidad del Valle. Disponible en: http://files.juancarlosrivera. webnode.com.co/200000011-d41ead517a/ Investigacion\%20contable.pdf

Parra-Moreno, Ciro (2002). Investigación-acción y desarrollo profesional. Revista Educación y Educadores, 5, 113-125. Disponible en: http://educacionyeducadores.unisabana. edu.co/index.php/eye/article/view/515/1602

Patiño-Jacinto, Ruth Alejandra\& Santos, Gerardo (2009). La investigación formativa en los programas de Contaduría Pública, caso Colombia. Capic Review, 7, 23-34. Disponible en: https://dialnet.unirioja.es/descarga/ articulo/3736534.pdf

Quiñones-Cárdenas, Jeremías \& VélezÁlvarez, Consuelo (2004). Algunas condiciones pedagógicas para la formación y el desarrollo de la investigación en la universidad. Actualidades Investigativas en Educación, 4 (1), 1-27. Disponible en: http://www.redalyc.org/pdf/447/44740103. pdf

Restrepo-Gómez, Bernardo (1997). Investigación en educación. Bogotá: Corcas Edites.

Disponible en: http://datateca.unad.edu.co/ contenidos/551075/2016-1/Unidad_1/03_ InvestigacionEducativa_BernardoRestrepo_ Unidades1-2-3.pdf

Restrepo-Gómez, Bernardo (2002). Conceptos y aplicaciones de la investigación formativa $y$ criterios para evaluar la investigación en sentido estricto. Bogotá: Consejo Nacional de Acreditación, CNA. Disponible en: http:// www.cna.gov.co/1741/articles-186502_doc_ academico5.pdf

Restrepo-Gómez, Bernardo (2003). Investigación formativa e investigación productiva de conocimiento en la universidad. Nómadas, 18, 195-202. Disponible en: https://www.ucentral. edu.co/images/editorial/nomadas/docs/ nomadas_18_18_inv_formativa.PDF

Ríos-León, Roberto Antonio (2013). La formación para la investigación de contadores públicos colombianos: un asunto de estrategia. Revista Científica General José María Córdova, 11 (11), 175-206. Disponible en: http://www.scielo.org.co/ pdf/recig/v11n11/v11n11a09.pdf Rojas-Betancur, Héctor Mauricio (2009). Formar investigadores e investigadoras en la universidad: optimismo e indiferencia juvenil en temas científicos. Revista Latinoamericana de Ciencia Sociales, Niñez y Juventud, 7 (2), 1595-1618. Disponible en: http://revistaumanizales.cinde.org.co/ 
index.php/Revista-Latinoamericana/article/ view/172/75

Ruiz-Bolívar, Carlos \& Torres-Pacheco, Virginia (2005). La enseñanza de la investigación en la universidad: el caso de una universidad pública venezolana. Investigación y Postgrado, 20 (2), 13-34. Disponible en: http://revistas.upel.edu.ve/index.php/ revinpost/article/view/1492/601

Sá, Creso M. (2008). Interdisciplinary Strategies in U.S. Research Universities. Higher Education: The International Journal of Higher Education and Educational Planning, 55 (5), 537-552.

Sancho-Gil, Juana María (2001). Docencia e investigación en la universidad: una profesión, dos mundos. Educar, 28, 41-60. Disponible en: http://www.raco.cat/index.php/ Educar/article/download/20742/20582

Tamayo y Tamayo, Mario (2005). El proceso de la investigación científica: incluye evaluación y administración de proyectos de investigación. México: Limusa.

Upegui-Velásquez, María Eugenia (2002).

La enseñanza de la contabilidad: por una investigación formativa mediante el aprendizaje cooperativo. Monografía para optar al título de especialista en didáctica universitaria, Medellín, Universidad de Antioquia. Disponible en: http://ayura.udea.edu.co:8080/jspui/ bitstream/123456789/256/1/JC0343.pdf
Valera-Alfonso, Orlando (1999). Orientaciones pedagógicas contemporáneas. Bogotá: Magisterio.

Weerts, David J. \& Sandmann, Lorilee R. (2010). Community Engagement and Boundary-Spanning Roles at Research Universities. The Journal of Higher Education, 81 (6), 632-657.

Zúñiga, Myriam (1998). El proyecto educativo institucional como nueva opción de vinculación entre las instituciones colombianas formadoras de docentes y las escuelas. En Organización de Estados Iberoamericanos para la Educación, la Ciencia y la Cultura, OEI. Una educación con calidad y equidad: Encuentro Internacional sobre Formación de Profesores de Educación Básica, 81-100. Madrid: OEI.

- Fecha de recepción: 17 de marzo de 2015

- Fecha de aceptación: 31 de agosto de 2015

- Disponible en línea: 30 de junio de 2016

\section{Para citar este artículo}

Avendaño-Castro, William Rodrigo; RuedaVera, Gerson \& Paz-Montes, Luisa Stella (2016). La investigación formativa en las prácticas docentes de los profesores de un programa de contaduría pública. Cuadernos de Contabilidad, 17(43), 157-182. http://dx.doi.org/10.11144/Javeriana.cc1743.ifpd 